Artigo Original

\title{
Relação entre independência e o nível de disfunção motora e funcional em pacientes hemiparéticos
}

\section{Relation between functional independence and motor impairments in hemiparetic post-stroke patients}

Camila Torriani ${ }^{1}$, Eliane Pires de Oliveira Mota², Sônia Hitomi Pedrosa Kazurayama ${ }^{3}$, Stella Ruhe Burin $^{3}$, Tatiana Mengatti ${ }^{3}$, Juliana Caminho ${ }^{3}$, Francyelle Bastos ${ }^{3}$, Graziela Bastos ${ }^{3}$, Jenifer Chris ${ }^{3}$.

\begin{abstract}
RESUMO
Objetivo: O objetivo deste estudo foi estabelecer a correlação entre a independência funcional, tempo de lesão e o nível de disfunção motora em pacientes hemiparéticos. Método: Foram selecionados aleatoriamente 23 pacientes com seqüelas de Acidente Vascular encefálico (AVE). Os critérios de exclusão foram: alteração cognitiva grave, afasia de compreensão e impossibilidade de participar do estudo. Após assinarem o termo de consentimento, os sujeitos foram submetidos a aplicação do questionário de Medida de Independência Funcional (MIF). Para aqueles que apresentavam afasia de expressão, a aplicação do questionário foi feita com o cuidador. As categorias foram agrupadas em seis dimensões: autocuidado, controle de esfíncteres, transferências, locomoção, comunicação, e cognição social. Avaliou-se a correlação dos itens da MIF com gênero, lado hemiparético e predomínio braquial, crural ou proporcionada do acometimento. Resultados: A variável gênero se correlacionou com o Controle de Esfincter e Cognição Social. As mulheres apresentaram maior Cognição Social do que os homens $(p=0,01)$ e os homens apresentaram tendência a maior controle do esfíncter do que as mulheres $(p=0,06)$. Comparando-se o Predomínio de acometimento corporal, observamos que o Predomínio Crural foi menor em relação aos demais predomínios Braquial e Proporcionada $(p=0,01)$ para Comunicação. Conclusão: Houve correlação entre disfunção motora e funcional para o item comunicação da MIF, e o acometimento com predomínio crural foi menor em relação aos demais predomínios.
\end{abstract}

\section{Unitermos: Hemiplegia, Acidente Cerebrovascular, Fisioterapia, Avaliação.}

Citação: Torriani C, Mota EPO, Kazurayama SHP, Burin SR, Mengatti T, Caminho J, Bastos F, Bastos G, Chris J. Relação entre independência e o nível de disfunção motora e funcional em pacientes hemiparéticos. Rev Neurocienc 2007;15(1):32-36.

\section{SUMMARY}

Objective: The purpose of this study is to verify the correlation between functional independence and motor impairments in hemiparetic post-stroke patients. Method: Twenty-three stroke patients were randomly selected. The exclusion criteria were severe cognitive dysfunction, comprehension aphasia and impossibility of participating. Before the patients signed the Consent Form, they were submitted to the Functional Independence Measure (FIM). The questionnaire has been applied with the caregivers for those who had expression aphasia. The categories were divided in 6 domains: take care of yourself, sphincter control, transference, locomotion, communication and social cognition. We evaluated the correlation of FIM with gender, hemiparetic side, and brachial, crural or combined impaired predomination. Results: Gender was correlated with sphincter control and social cognition. The women presented more Social Cognition than men did $(p=0.01)$ and the men presented a tendency in more sphincter control than women did $(p=0.06)$. Comparing impairment predomination, we observed that there was less

Trabalho realizado na UniFMU, São Paulo.

1.Mestranda em Biodinâmica do movimento USP. Docente e supervisora de estágio do setor Neuro Adulto da UniFMU. Docente dos cursos de pós graduação na UNICID, GAMA FILHO, FMU.

2. Mestranda em Educação UNICID. Docente e supervisora de estágio do setor Neuro Adulto UniFMU.

3. Acadêmicos do 40 ano do curso de Fisioterapia da UniFMU.

Endereço para Correspondência: Camila Torriani, Av. Santo Amaro, 1329, Vila Nova Conceição - CEP 0451 1-001, São Paulo-SP Email: camilatorriani@uol.com.br

Trabalho recebido em 02/10/2006 • Revisão: de 01/10/2006 a 29/11/2006 • Aprovado em 30/11/2006 • Conflito de interesses: não 
crural one that brachial and combined $(p=0.01)$ related to communication. Conclusion: There was a correlation between motor and functional impairment and the FIM communication item, and there was less crural impairment than the other predominancies.

\section{Keywords: Hemiplegia, Stroke, Physical Therapy, Evaluation.}

Citation: Torriani C, Mota EPO, Kazurayama SHP, Burin SR, Mengatti T, Caminho J, Bastos F, Bastos G, Chris J. Relation between functional independence and motor impairments in hemiparetic post-stroke patients. Rev Neurocienc 2007;15(1):32-36.

\section{INTRODUÇÃO}

O acidente vascular encefálico (AVE) tem como definição sinais e sintomas neurológicos de ocorrência súbita, com perda de funções encefálicas focais ou generalizadas, de origem vascular, com duração superior a vinte e quatro horas ou levando a óbito. Aproximadamente $80 \%$ dos AVE's são causados por um baixo fluxo sanguíneo cerebral (isquemia) e outros $20 \%$ por hemorragias tanto intraparenquimatosas como subaracnóideas ${ }^{1}$.

O envelhecimento da população brasileira advém de alterações fisiológicas que podem estar associadas a doenças crônicas e degenerativas, conduzindo a déficits funcionais e declínio da morbidade. As evidências clínicas demonstram que a incidência de AVE no Brasil é alta, principalmente em indivíduos idosos, estando associada a uma alta taxa de sobrevivência que declina levemente com o avanço da idade, de $79 \%$ dos 75 aos 84 anos para $67 \%$ acima dos 85 anos. Com o envelhecimento populacional brasileiro, estima-se que a prevalência do AVE aumente nessa população?

O AVE evolui com manifestações clínicas e comumente alterações motora e sensitiva, prejudicando a função física. Há também alterações nas funções cognitiva, perceptiva, visual, emocional e continência que podem estar associados a severidade do quadro clínico dependerá, que dependerá também da extensão da lesão. A presença de déficit do controle motor pode ser caracterizada por fraqueza, alteração de tônus e movimentos estereotipados, que podem limitar as habilidades para realizar atividades como deambular, subir escadas e autocuidar-se².

No déficit neurológico focal do AVE depende do tamanho, localização da lesão e da quantidade de fluxo sanguíneo colateral. Os déficits neurológicos unilaterais são resultados da interrupção do sistema carotídeo, e os déficits neurológicos bilaterais são resultantes da interrupção do suprimento vascular basilar. As artérias que podem estar acometidas são artérias cerebrais anterior, média e posterior, cujas alterações mais evidentes são respectivamente tronco e membro inferior, membro superior e alteração visual ${ }^{3}$.

A partir destes dados e pela incapacidade proporcionada pela doença é necessário analisar como estes pacientes desempenham suas atividades diárias para que um programa terapêutico específico possa ser traçado para cada paciente. Desta forma, o Sistema FIM (Functional Independence Measure) ou Sistema MIF (Medidas de Independência Funcional) como é chamado aqui no Brasil, classifica e registra o grau de independência funcional. O objetivo deste sistema é dar ênfase à dificuldade e a limitação do paciente, com o entendimento sobre a capacidade de adaptação que se pode observar na realização de tarefas, algumas vezes complexas, do cotidiano ${ }^{4}$.

A especificidade deste sistema deve ter a assistência ao paciente portador de lesão incapacitante e de doenças crônico-degenerativas. Seu objetivo primordial é avaliar de forma quantitativa a carga de cuidados demandada por uma pessoa para a realização de uma série de tarefas motoras e cognitivas de vida diária. Entre as atividades avaliadas estão os autocuidados, transferências, locomoção, controle esfincteriano, comunicação e cognição social, que inclui memória, interação social e resolução de problemas. Cada uma dessas atividades é avaliada e recebe uma pontuação que parte de 1 (dependência total) a 7 (independência completa), assim a pontuação total varia de 18 a 126. Estão descritos dois domínios na MIF, o motor e o cognitivo.

Por isso, é importante a análise das atividades de vida diária nos pacientes portadores de AVE correlacionando com o tempo de lesão com o hemicorpo acometido e o predomínio da disfunção (braquial ou crural), o que possibilitará maior entendimento desta população e consequentemente reabilitação adequada.

Estabelecer a correlação entre a independência funcional, o tempo de lesão e o nível de disfunção motora em pacientes hemiparéticos à direita e esquerda.

\section{MÉTODO}

Foram selecionados aleatoriamente 23 pacientes com seqüelas de AVE que se encontram em atendimento no setor de Fisioterapia Neurológica Adulto, no Centro Universitário UniFMU. Os critérios de exclusão foram: alterações cognitivas graves, afasia de compreensão e impossibilidade de participar do estudo. Os pacientes que não apresentassem tais caracte- 
rísticas e tivesse disponibilidade para participar do estudo foi incluído.

O estudo foi aprovado por Comitê Ético interno da Instituição, sendo que foram respeitados os aspectos éticos concernentes a Resolução de no 196 de 10 de outubro de 1996, que delimita diretrizes e normas regulamentadoras de pesquisas envolvendo seres humanos. A coleta de dados iniciou-se após assinatura de termo de Consentimento Livre e esclarecido contendo explicações detalhadas sobre o estudo e sua finalidade.

Após preencherem o termo de consentimento os sujeitos foram submetidos à aplicação do questionário de Medida de Independência Funcional (MIF) sendo que aqueles que apresentavam afasia de expressão, a aplicação do questionário foi feita com o cuidador. 0 instrumento MIF avalia 18 categorias pontuadas de um a sete e classificadas quanto ao nível de dependência para a realização da tarefa. As categorias são agrupadas em seis dimensões: autocuidado, controle de esfíncteres, transferências, locomoção, comunicação e cognição social. Cada dimensão é analisada pela soma de suas categorias referentes. Assim, quanto menor a pontuação, maior é o grau de dependência. Somando-se os pontos das dimensões da MIF obtémse um escore total mínimo de 18 e o máximo de 126 pontos, que caracterizam os níveis de dependência pelos sub-escores.

Na versão brasileira da MIF uma boa equivalência cultural e boa reprodutibilidade, quando aplicado em 164 pacientes por dois examinadores distintos, e reavaliados por apenas um desses examinadores após uma semana (teste/reteste)5. A validade convergente da MIF pode ser observada tanto para as tarefas motoras em pacientes com Lesão Medular como para os pacientes com Lesão Encefálica. A versão brasileira da MIF mostrou-se sensível a alterações e clinicamente útil para a avaliação de resultados de reabilitação em pacientes ambulatoriais subagudos e crônicos no Brasil ${ }^{6}$.

\section{Análise Estatística}

O teste de Spearman foi usado para avaliar se havia correlação entre a independência funcional e as variáveis gênero, lado hemiparético (direito e esquer- do), disfunção motora e predomínio braquial, crural ou proporcionada do acometimento. Os resultados foram obtidos através dos testes não paramétricos de Kruskal-Wallis e Wann-Whitney.

Para determinarmos o grau da correlação entre as variáveis foi utilizada a escala de classificação: péssima (0-20\%), ruim (20-40\%), regular (40-60\%), boa (60-80\%) e ótima (80-100\%).

\section{RESULTADOS}

A variável gênero se correlacionou com o desempenho nas MIFs Controle de Esfincter $(p=0,05)$ e Cognição Social $(0,009)$. As variáveis lado hemiparético e predomínio de acometimento (braquial e crural) não se correlacionaram com as MIFs (Tabela 1). Comparando-se os gênero, observamos que as mulheres apresentaram maior Cognição Social do que os homens $(p=0,01)$ e os homens apresentaram tendência a maior controle do esfíncter do que as mulheres $(p=0,06)$ (Tabela 2). Observamos que não houve diferença significante quando comparamos o lado hemiparético e as MIFs (Tabela 3). Comparandose o Predomínio de acometimento corporal (braquial, crural e proporcionada) observamos que o Predomínio Crural foi menor em relação aos demais predomínios Braquial e Proporcionada $(p-0,01)$ para Comunicação (Tabelas 4 e 5).

\section{DISCUSSÃO}

Nos achados deste estudo não foram encontradas diferenças entre independência funcional e hemiparéticos à direita e esquerda.

Estes achados não corroboram com outros estudos que verificou que pacientes com hemiplegia à direita podem ser cautelosos e desorganizados na solução de uma determinada tarefa, e pacientes com hemiplegia à esquerda tendem a ser rápidos e impulsivos e aparentemente não cientes dos déficits presentes. Sugerindo que paciente com hemiplegia à direita ou esquerda, apresentam déficits comportamentais, motores e intelectuais, que interferem proporcionalmente

Tabela 1. Correlação entre os itens do Medida de Independência Funcional e as variáveis sexo, lado hemiparético e predomínio de acomentimento:

\begin{tabular}{ccccccc}
\hline \multirow{2}{*}{ Correlação } & \multicolumn{2}{c}{ Sexo } & \multicolumn{2}{c}{ Lado Hemi } & \multicolumn{2}{c}{ Predomínio } \\
\cline { 2 - 7 } & Corr. & p-valor & Corr. & p-valor & Corr. & p-valor \\
\hline Auto Cuidado & $-22,3 \%$ & 0,30 & $18,7 \%$ & 0,39 & $-19,3 \%$ & 0,38 \\
\hline Controle Esfincter & $40,7 \%$ & 0,05 & $-5,2 \%$ & 0,81 & $4,9 \%$ & 0,82 \\
\hline Mobilidade & $2,7 \%$ & 0,90 & $-1,4 \%$ & 0,95 & $-1,0 \%$ & 0,96 \\
\hline Locomoção & $-22,7 \%$ & 0,29 & $14,8 \%$ & 0,50 & $-16,9 \%$ & 0,44 \\
\hline Comunicação & $-8,3 \%$ & 0,70 & $13,5 \%$ & 0,54 & $-9,1 \%$ & 0,68 \\
\hline Cognição Social & $-53,1 \%$ & 0,009 & $-9,1 \%$ & 0,68 & $-15,4 \%$ & 0,48 \\
\hline Total & $-22,2 \%$ & 0,31 & $12,4 \%$ & 0,57 & $-16,8 \%$ & 0,44 \\
\hline
\end{tabular}


Tabela 2. Comparação dos valores do Medida de Independência Funcional com a variável sexo (feminino-fem e masculino-masc):

\begin{tabular}{|c|c|c|c|c|c|c|c|c|c|}
\hline Sexo & & Média & Mediana & $\begin{array}{l}\text { Desvio } \\
\text { Padrão }\end{array}$ & Quartil 1 & Quartil 3 & Tamanho & IC & p-valor \\
\hline \multirow{2}{*}{ Auto Cuidado } & Fem & 32,7 & 39 & 10,9 & 27 & 40 & 9 & 7,1 & \multirow{2}{*}{0,29} \\
\hline & Masc & 29,3 & 33,5 & 9,9 & 20 & 37 & 14 & 5,2 & \\
\hline \multirow{2}{*}{ Controle Esfincter } & Fem & 10,9 & 13 & 3,9 & 10 & 13 & 9 & 2,5 & \multirow{2}{*}{0,06} \\
\hline & Masc & 12,5 & 14 & 3,2 & 13,2 & 14 & 14 & 1,7 & \\
\hline \multirow{2}{*}{ Mobilidade } & Fem & 14,6 & 18 & 7,4 & 11 & 20 & 9 & 4,8 & \multirow{2}{*}{0,89} \\
\hline & Masc & 15,1 & 18,5 & 6,6 & 8,2 & 20 & 14 & 3,4 & \\
\hline \multirow{2}{*}{ Locomoção } & Fem & 10,9 & 14 & 4,4 & 8 & 14 & 9 & 2,9 & \multirow{2}{*}{0,29} \\
\hline & Masc & 9,7 & 12 & 4,3 & 7 & 13 & 14 & 2,3 & \\
\hline \multirow{2}{*}{ Comunicação } & Fem & 21,4 & 21 & 2,9 & 21 & 22 & 9 & 1,9 & \multirow{2}{*}{0,69} \\
\hline & Masc & 20,6 & 21,5 & 2,6 & 18,5 & 22 & 14 & 1,4 & \\
\hline \multirow{2}{*}{ Cognição Social } & Fem & 19,4 & 20 & 2,1 & 19 & 21 & 9 & 1,4 & \multirow{2}{*}{0,01} \\
\hline & Masc & 16,3 & 16 & 3,4 & 13,5 & 19,7 & 14 & 1,8 & \\
\hline \multirow{2}{*}{ Total } & Fem & 109,9 & 123 & 27,1 & 98 & 127 & 9 & 17,7 & \multirow{2}{*}{0,29} \\
\hline & Masc & 103,4 & 115 & 23,9 & 81,7 & 121 & 14 & 12.5 & \\
\hline
\end{tabular}

Tabela 3. Comparacao dos valores da Medida de Independência Funcional com a variavel lado hemiparetico (direito-dir e esquerdo-esq):

\begin{tabular}{|c|c|c|c|c|c|c|c|c|c|}
\hline Lado Hemi & & Média & Mediana & $\begin{array}{l}\text { Desvio } \\
\text { Padrão }\end{array}$ & Quartil 1 & Quartil 3 & Tamanho & IC & p-valor \\
\hline \multirow{2}{*}{ Auto Cuidado } & Dir. & 29,1 & 32 & 10,4 & 19,5 & 38 & 15 & 5,2 & \multirow{2}{*}{0,38} \\
\hline & Esq. & 33,4 & 38 & 10,0 & 32 & 40 & 8 & 6,9 & \\
\hline \multirow{2}{*}{ Controle Esfincter } & Dir. & 12,1 & 14 & 3,4 & 12 & 14 & 15 & 1,7 & \multirow{2}{*}{0,81} \\
\hline & Esq. & 11,5 & 13,5 & 3,8 & 10 & 14 & 8 & 2,7 & \\
\hline \multirow{2}{*}{ Mobilidade } & Dir. & 14,5 & 18 & 7,3 & 8,5 & 21 & 15 & 3,7 & \multirow[b]{2}{*}{0,95} \\
\hline & Esq. & 15,6 & 19 & 6,0 & 12,5 & 20 & 8 & 4,2 & \\
\hline \multirow{2}{*}{ Locomoção } & Dir. & 9,6 & 12 & 4,4 & 6 & 14 & 15 & 2,2 & \multirow[b]{2}{*}{0,49} \\
\hline & Esq. & 11,2 & 13 & 4,1 & 10,7 & $\frac{14}{14}$ & 8 & $\begin{array}{l}2, L \\
2,8\end{array}$ & \\
\hline \multirow{2}{*}{ Comunicação } & Dir. & 20,7 & 21 & 2,4 & 19,5 & 22 & 15 & 1,2 & \multirow{2}{*}{0,53} \\
\hline & Esq. & 21,4 & 22 & 3,3 & 19,5 & 22 & 8 & 2,3 & \\
\hline \multirow{2}{*}{ Cognição Social } & Dir. & 17,8 & 19 & 3,2 & 16 & 20 & 15 & 1,6 & \multirow{2}{*}{0,67} \\
\hline & Esq. & 17,0 & 17,5 & 3,6 & 14,5 & 20 & 8 & 2,5 & \\
\hline \multirow{2}{*}{ Total } & Dir. & 103,7 & 116 & 24,9 & 78,5 & 122 & 15 & 12,6 & \multirow{2}{*}{0,56} \\
\hline & Esq. & 110,1 & 121 & 25,7 & 106,5 & 126 & 8 & 17,8 & \\
\hline
\end{tabular}

na independência funcional de ambos ${ }^{4}$.

Pacientes com hemiplegia/paresia à esquerda costumam apresentar-se com diminuição no comprometimento na comunicação e, por isso, tem melhor desempenho funcional. Por outro lado, o domínio cognitivo da MIF apresentou pontuação associada ao tipo de deficiência decorrente da lesão encefálica. As lesões no hemisfério direito resultaram classicamente em hemiparesia à esquerda e comprometimento cognitivo na forma de negligência visual, tátil ou motora à esquerda, além de dificuldade de percepção das próprias incapacidades. Porém, de forma geralmente menos intensa que nas lesões a direita, nas quais as limitações envolvem a comunicação de forma mais grave, comprometimento da memória e dificuldade para acompanhar instruções e treinamentos. Desta forma, espera-se que os pacientes com lesão no hemisfério direito tenham perfis de independência funcionais melhores que os demais, resultado este que em nosso estudo não pôde ser observado?

Além destas distinções entre lesão encefálica à direita e lesão à esquerda, Haaland e Harrington descrevem que pacientes com lesão à direita apresentam alterações que são estritamente no hemicorpo contralateral, enquanto que pacientes com lesão no hemisfério esquerdo apresentam déficits motores que podem ser não somente no hemicorpo contralateral à lesão, mas também ipsilateralmente. Além disso, o hemisfério cerebral esquerdo apresenta maior atuação no planejamento motor, fato este que também corrobora ao fato de pacientes hemiplégicos à direita apresentarem maiores dificuldades em seqüências funcionais diárias. Estes fatos nos levam a inferir que pacientes com lesão encefálica à esquerda têm maior potencial para prejuízos funcionais ${ }^{8-10}$.

Um outro fator, não investigado neste estudo, mas que merece atenção na repercussão funcional nos pacientes é o fato de o lado corporal acometido ser o lado dominante. Pois, pacientes com hemiplegia/paresia no hemicorpo cuja mão era dominante antes da lesão apresentam menores prejuízos funcionais que os pacientes com lesão no hemicorpo cuja mão não era dominante. Desta forma, sugerimos para um próximo estudo a inclusão desta variável na análise funcional destes pacientes ${ }^{11}$.

Verificou-se também, que no item cognição social o gênero feminino apresentou melhor resultado quando comparado ao masculino, o que sugere que o gênero é um fator que influenciou neste resultado, talvez porque as mulheres apresentem mais facilidade para interagir socialmente, porém não foi encontrado na literatura um fator determinante para tal resultado.

Outro achado relevante do nosso estudo foi encontrar maior comprometimento da cognição social em pacientes com predomínio crural, sendo talvez possível justificá-lo pelo fato que pacientes com comprometi- 
Tabela 4. Comparacao de valores da Medida de Independência Funcional com variável de acometimento (braquial, crural e proporcionada):

\begin{tabular}{|c|c|c|c|c|c|c|c|c|c|}
\hline \multicolumn{2}{|c|}{ Predomínio } & Média & Mediana & $\begin{array}{l}\text { Desvio } \\
\text { Padrão }\end{array}$ & Quartil 1 & Quartil 3 & Tamanho & IC & p-valor \\
\hline \multirow{3}{*}{ Auto Cuidado } & Braquial & 33,8 & 36,5 & 7,8 & 30,7 & 40 & 12 & 4,4 & \multirow{3}{*}{0,28} \\
\hline & Crural & 24,4 & 16 & 12,9 & 15 & 37 & 5 & 11,3 & \\
\hline & Proporcionada & 29,3 & 29,5 & 11,2 & 20,5 & 38,5 & 6 & 8,9 & \\
\hline \multirow{3}{*}{ Controle Esfincter } & Braquial & 12,7 & 13,5 & 1,9 & 12,2 & 14 & 12 & 1,1 & \multirow{3}{*}{0,25} \\
\hline & Crural & 8,2 & 8 & 5,8 & 3 & 14 & 5 & 5,1 & \\
\hline & Proporcionada & 13,3 & 14 & 1,2 & 13,2 & 14 & 6 & 0,9 & \\
\hline \multirow{3}{*}{ Mobilidade } & Braquial & 16,4 & 18,5 & 4,7 & 13,2 & 20 & 12 & 2,6 & \multirow{3}{*}{0,69} \\
\hline & Crural & 10,6 & 5 & 9,5 & 3 & 21 & 5 & 8,3 & \\
\hline & Proporcionada & 15,3 & 19 & 7,4 & 10,5 & 20,7 & 6 & 5,9 & \\
\hline \multirow{3}{*}{ Locomoção } & Braquial & 11,2 & 13 & 3,7 & 10,7 & 14 & 12 & 2,1 & \multirow{3}{*}{0,67} \\
\hline & Crural & 8,4 & 8 & 5,5 & 3 & 14 & 5 & 4,8 & \\
\hline & Proporcionada & 9,5 & 9,5 & 4,5 & 7 & 13,5 & 6 & 3,6 & \\
\hline \multirow{3}{*}{ Comunicação } & Braquial & 21,7 & 22 & 2,9 & 20 & 22 & 12 & 1,6 & \multirow{3}{*}{0,01} \\
\hline & Crural & 18,0 & 17 & 2,0 & 17 & 19 & 5 & 1,7 & \\
\hline & Proporcionada & 21,7 & 22 & 0,5 & 21,2 & 22 & 6 & 0,4 & \\
\hline \multirow{3}{*}{ Cognição Social } & Braquial & 17,7 & 20 & 4,0 & 14,2 & 21 & 12 & 2,3 & \multirow{3}{*}{0,51} \\
\hline & Crural & 17,0 & 17 & 2,0 & 15 & 19 & 5 & 1,7 & \\
\hline & Proporcionada & 17,5 & 18 & 2,9 & 16 & 20 & 6 & 2,4 & \\
\hline \multirow{3}{*}{ Total } & Braquial & 113,7 & 119 & 18,8 & 110 & 126,2 & 12 & 10,7 & \multirow{3}{*}{0,19} \\
\hline & Crural & 86,6 & 69 & 32,6 & 62 & 121 & 5 & 28,5 & \\
\hline & Proporcionada & 106,7 & 111 & 23,7 & 88,2 & 124,7 & 6 & 19,0 & \\
\hline
\end{tabular}

Tabela 5. Comparação do valor da Medida de Independência Funcional no itens comunicacao com variavel de acometimento (braquial, crural e proporcionada)

\begin{tabular}{ccc}
\hline Comunicação & Braquial & Crural \\
\hline Crural & $0,01^{\star}$ & \\
\hline Proporcionada & 0,76 & $0,008^{*}$ \\
\hline
\end{tabular}

mento crural, tem o convívio social mais difícil, devido à dependência de cadeira de rodas para locomover-se, ao passo que pacientes com predomínio braquial apresentam menos dificuldade de locomoção proporcionando uma interação social mais acessível.

\section{CONCLUSÃO}

Não foi estabelecida correlação entre a independência funcional e o nível de acometimento motor. Houve correlação entre predomínio crural da hemiplegia e o item comunicação na MIF. O gênero feminino apresentou melhor cognição social e não houve diferença entre a independência funcional e o lado da hemiparesia/ plegia.

Sugerem-se novos estudos visando aumentar a amostra, torná-la mais homogênea e controlar as variáveis intervenientes a fim de estabelecer quais fatores afetam realmente a independência funcional na hemiparesia/ plegia.

\section{REFERÊNCIAS BIBLIOGRÁFICAS}

1. Neto AC. Acidente Vascular Cerebral - Neurology, v8, São Paulo: Revinter. 2001, p1-13

2. Fuscaldi L, Silva CS, Augusto AC, Souza AC, Goular F, Lima MR. Fortalecimento muscular e condicionamento físico em hemiplégicos. Acta Fisiatr 2000; 7(3):108-118.

3. Umphred D. A Fisioterapia Neurológica. $2^{\circ}$ edição. São Paulo: Editora Manole,1994, p615-656.

4. Moura RMF, Lima RCM, Lage DC, Amaral EAA. Efeitos do treinamento aeróbio na qualidade de vida e na capacidade funcional de indivíduos hemiparéticos crônicos. Acta Fisiatr 2005; 12(3): 8-13.

5. Riberto M, Miyazaki MH, Filho DJ, Sakamoto H, Battistella LR. Reprodutibilidade da versão brasileira da Medida de Independência Funcional. Acta Fisiatr 2001; 1(8):45-52.

6. Riberto M, Miyazaki MH, Jucá SSH, Sakamoto H, Pinto PPN, Battistella LR.
Validação da Versão Brasileira da Medida de Independência Funcional. Acta Fisiatr 2004; 2(11):72-76.

7. Riberto M, Pinto PPN, Sakamoto H, Battistella LR. Independência funciona de pacientes com lesão medular Acta Fisiatr 2005; 12(2): 61-66.

8. Haaland KY, Harrington DL. Limb-sequencing déficits after left but not right hemisphere damage. Brain and Cognition 2004; 24: 104-122.

9. Sabate M, Gonzalez B, Rodriguez M. Brain lateralization of motor imagery: motor planning asymmetry as a cause of movement lateralization. Neuropsychologia 2004; 42: 1041-1049.

10. Luft AR, Walter S, Forrester L, Smith GV, Whitall J, Macko RF, et al. Lesion location alters brain activation in chronically impaired stroke survivors. Neuroimage 2004; 21:924-935

11. Harris JE, Eng JJ. Individuals with dominant hand affected following stroke demostrate less impairment than those with the nondominant hand affected. Neurorehabil S Jouvet Neural Repair 2006; 20(3): 380-389. 